\title{
Itinéraire erratique et mutation identitaire dans Desirada de M. Condé
}

\author{
Erratic itinerary and identity mutation \\ in M. Condé's Desirada
}

\author{
Pierre Suzanne Eyenga Onana \\ U niversité de Yaoundé I \\ petereyenga@gmail.com
}

\begin{abstract}
How does the phenomenon of immigration cause migrants to change their psychological, socio-professional and cultural identities to such an extent that they become hybrids? Based on Henri Mitterand's sociocriticism, this study is organized in three parts. First, we show how immigration proves to be a stumbling block in the migrant's identity-building process, as well as a springboard for reinventing oneself in the foreign space. Then we look at the aesthetic dimension that allows us to grasp the novel above all as a work of art and not a history textbook. Finally, we illustrate that identity, in Condé's case, results from the sum of the cultures that the migrant encounters throughout his or her erratic itinerary.
\end{abstract}

\section{Keywords}

erratic itinerary, migrant, mutation, identity, sociocriticism.

\begin{abstract}
Resumen
¿De qué manera el fenómeno de la inmigración hace que los migrantes cambien su identidad psicológica, socioprofesional y cultural hasta el punto de convertirse en seres híbridos? Basado en la sociocrítica de Henri Mitterand, este estudio está organizado en tres partes. En primer lugar, mostramos cómo la inmigración resulta ser un escollo en el proceso de construcción de la identidad del migrante, así como un trampolín para reinventarse en el espacio extranjero. A continuación, observamos la dimensión estética que nos permite captar la novela principalmente como una obra de arte y no como un libro de texto de historia. Por último, ilustramos que la identidad, en la Desirada de Condé, resulta de la suma de las culturas que el migrante encuentra a lo largo de su errático itinerario.
\end{abstract}

\section{Palabras clave}

itinerario errático, migrante, mutación, identidad, sociocrítica. 


\section{Introduction}

La notion d'immigration renvoie, selon le Dictionnaire Larousse Encyclopédique, à "l'arrivée dans un pays d'étrangers venus s'y installer et y travailler" (2008: 44). Le sens de ce concept se trouve complété à travers la définition qui l'établit comme "l'action de s'installer et de travailler dans un pays étranger pour une assez longue durée, voire définitivement" (Aprile \& Dufoix, 2009: 185). La problématique de l'immigration reste au cœur de l'actualité mondiale, qu'elle soit intracontinentale ou extracontinentale. De tout temps, l'humanité reste marquée par le déplacement des hommes, d'un espace vers un autre, et ce pour une raison ou une autre. Une chose est sûre toutefois, "l'homme naît du voyage, son corps comme son esprit sont façonnés par le nomadisme. Le propre de l'homme c'est d'abord la course d'un bipède" (Attali, 2003: 8). De sorte que le phénomène du déplacement s'inscrit dans le quotidien de l'homme. Les nombreux projets de déplacements d'Africains vers l'Europe, notamment en France, en Espagne ou en Italie, traduisent dans les faits cette volonté de résoudre autrement la question de son inconfort social dans son cadre de vie originel. Ceci explique pourquoi, au XVII ${ }^{\text {ème }}$ siècle, la découverte de nouvelles parties du monde entretient des mythes prospectifs d'un Eldorado à conquérir. Mais par-delà cette quête effrénée d'un ailleurs meilleur, la problématique de l'immigration génère de nouveaux paradigmes sémantiques à l'instar de la question de l'identité.

Question omniprésente depuis l'histoire de la pensée, l'identité semble trouver son fondement depuis la célèbre allégation socratique homme, connais-toi toi-même et tu connấtras l'univers et les dieux. La Négritude, née en France dans les années 1930 sous l'impulsion de Césaire, Damas et Senghor, entre autres, autant que le féminisme, et plus proche de nous la créolité, sont autant de moments phares de l'Histoire du monde qui montrent la nécessité de s'affirmer et d'affirmer son étiquette particulière à la face du monde. En ces temps-là, les Noirs s'estimaient honnis et méprisés à cause de la couleur de leur peau. Les femmes exigeaient l'écho de leur voix dans le concert des débats de la cité. La femme combattait une société phallocratique qui mettait ses droits sous le boisseau. Mais ces combats ne sont pas achevés à ce jour même s'ils ont parfois changé de cibles ou se sont renouvelés au niveau de leurs thématiques. Affirmant quant à eux leur statut singulier sous la forme d'un éloge, les Créoles jettent un pavé dans la mare en affichant leur identité composite de façon péremptoire: "ni Européens, ni Africains, ni Asiatiques, nous nous proclamons Créoles. Cela sera pour nous une attitude intérieure, mieux: une vigilance, ou [...], une sorte d'enveloppe mentale au mitan de laquelle se bâtira notre monde en pleine conscience du monde" (Bernabé, Chamoiseau \& Confiant, 1989:13). Cette assertion permet de comprendre que l'homme s'est toujours interrogé sur son identité, sa nature, son origine même voire sa destinée, en vue de savoir réellement qui il est. A l'image de l'homme qui marche sans cesse pour découvrir des cieux toujours neufs d'un éventuel mieux être, ainsi l'identité évolue-t-elle, dans l'espace et 
dans le temps. Elle se veut donc constructive en tant qu'elle est une disposition de l'esprit. Nadine Dolby (2006) traduit cette idée lorsqu'elle appréhende l'identité comme un mouvement perpétuel de structuration et de mutation de pouvoirs constants qui a lieu au sein d'une matrice globale ou locale. L'identité étant un indicateur de stabilité chez un individu, on s'interroge sur les facteurs qui entrainent la mutation identitaire chez les immigrés au cours de leurs pérégrinations. Comment, au prisme des formes esthétiques, le personnage migrant se départit-il d'un “ici” pour convoiter un "ailleurs"? Quels sont les enjeux idéologiques du phénomène de l'immigration dans nos sociétés à l'aune de la vision du monde que charrie Desirada?

La démarche sociocritique nous sert de grille d'analyse dans ce travail. Elle accorde un point d'honneur au critère d'immanence parce qu'elle "vise d'abord le texte. Elle est même lecture immanente en ce sens qu'elle reprend à son compte cette question du texte élaborée par la critique formelle et l'avalise comme objet d'étude prioritaire" (Duchet, 1979: 7). Desirada de Condé se situe donc au cœur de l'analyse. Mais le critère d'immanence que polarise la sociocritique n'occulte pas sa dimension éclectique. Voilà pourquoi la sociocritique s'ouvre aux autres sciences humaines par le travail de l'écriture ainsi que le souligne Mitterand: "le texte de roman ne se limite pas à exprimer un sens déjà là; par le travail de l'écriture, il produit un autre sens, il modifie l'équilibre antérieur du sens, il réfracte et transforme, tout à la fois, le discours social" (1980: 7).

\section{De la sociogenèse de l'immigration}

Epicentre social d'un événement ou d'un phénomène, la sociogenèse évoque les réalités socio-historiques qui favorisent le flux migratoire. Le roman revêt ainsi tout son sens de “chronique sociale" (Goldmann, 1964: 30), dès lors qu'il porte sur l'étude des aspects de la vie sociale, politique et culturelle qui incitent les jeunes à la révolte. C'est cette révolte qui, en s'amplifiant, résout la jeunesse à regarder ailleurs. L'examen de la sociogenèse porte sur le chaos social manifeste dans l'œuvre, les dérapages assortis au système éducatif et la critique de quelques notions inhérentes à l'immigration.

\subsection{Le chaos social}

Il est caractérisé par une situation de précarité existentielle exacerbée qui se voit dans le texte à travers des indices tels que le chômage, la corruption, la misère, les déviances de toutes sortes, notamment la prostitution, l'escroquerie, le banditisme et la pédophilie. Nous mettons en exergue le cas de la réclusion, de la pauvreté et de l'exclusion sociale.

La réclusion désigne le repli sur soi; elle renvoie à une vie d'autarcie, pénible à supporter du fait qu'elle n'ouvre pas des perspectives nouvelles pour l'avenir. La réclusion revêt 
deux formes: physique et psychologique. Elle est physique lorsque l'espace est isolé et s'assimile à un carcan. Peu ouvert, cet espace barricade tout plan et le personnage s'y retrouve enfermé. Confiné dans ce monde immonde, le personnage éprouve ici le désir de s'ouvrir sur l'environnement qui l'étouffe. Dans sa forme psychologique, la réclusion s'exhibe comme un sentiment de solitude intérieure induisant une méconnaissance de certaines réalités du monde. De la manière qu'elle se solde au plan physique, la réclusion psychologique suscite chez le personnage le besoin de liberté, l'envie de découvrir l'ailleurs. Le micro-espace de la Désirade en Guadeloupe convient bien à la description qu'on vient de faire. Il se présente comme un lieu isolé où les personnages se sentent renfermés. Dans de telles conditions, lesdits personnages cherchent à s'en affranchir: ils ne peuvent qu'envisager de partir ailleurs.

Tel est le cas pour Reynalda Altamira. Mère célibataire à l'âge de quatorze ans, elle abandonne sa fille Marie-Noëlle à La Pointe aux bons soins de sa marraine Ranélise et part en France. Dix ans après, elle fait venir Marie-Noëlle en France où elle se trouve et lui explique les raisons de son départ. Mue par le désir d'échapper à un environnement hostile qui la rejette, elle ne pouvait que se battre autrement. Le narrateur précise que: "Personne ne s'occupait de Reynalda, pas plus que de ces lépreux que, dans le temps, on parquait à la Désirade. Elle aurait pu devenir un des souffre-douleur du Dubouchage qui en comptait beaucoup si elle n'avait pas préparé son certificat d'étude dans la classe de Mme Lépervier' (Condé, 1997: 85). Si d'une part, cette citation soulève le problème de l'irresponsabilité parentale, elle montre surtout une jeunesse délaissée à son sort. Faute de moyens financiers pour l'encadrement des enfants, les parents démissionnent. Il se pose alors la nécessité pour les personnages reclus de mettre en place des stratégies diverses afin de résoudre un autre problème qu'engendre leur réclusion: celui de la pauvreté. Dans Le Ventre de l'Atlantique, Salie assimile la pauvreté à l'enfer en affirmant, sur un ton emphatique, que "la pauvreté, c'est la face visible de l'enfer, mieux vaut mourir que rester pauvre" (Diome, 2005: 30). La pauvreté qui frappe les personnages de M. Condé justifie leur départ vers d'autres cieux prometteurs. Ce fléau est de deux ordres: matériel et intellectuel.

Au plan matériel, la pauvreté se voit dans l'anarchie des constructions d'habitation, à l'accoutrement des jeunes insulaires, notamment celui du personnage du vieux pêcheur, ou de Reynalda pendant son enfance à la Désirade et à La Pointe. Le restaurant que gère Ranélise pour subvenir aux besoins de Marie-Noëlle présente peu d'allure. De même, la maison qu'habite Gian Carlo Coppini et sa famille dès leur arrivée à la Guadeloupe est en mauvais état. On peut aussi évoquer le domicile des insulaires présenté sous la forme de maisons en toit de chaume. La pauvreté trouve aussi son ancrage dans le chômage. C'est ce fléau qui pousse Ludovic, Stanley et sa bande de musiciens à quitter les Antilles pour la conquête des États-Unis ou de la France.

Au plan intellectuel, la pauvreté s'identifie à la carence d'instruction dont souffrent les pauvres. Ayant détecté le piège de l'analphabétisme qui se refermait sur elle, Reynalda relaie 
par ces mots ses prédispositions intellectuelles dénichées par l'évêque: "l'évêque était d'avis que je ne devais pas rester à la Désirade à manger de la misère, mais que je devais venir à La Pointe où il y avait tellement de possibilités d'avancement pour les nègres intelligents" (Condé, 1997: 72). Seules Reynalda et sa fille parviennent à poursuivre leurs études en terre étrangère. Toutefois, la difficulté à s'inscrire dans une école étrangère les expose à l'exercice de petits métiers tels que le gardiennage, la conduite et la musique profane. L'une des motivations de départ pour l'ailleurs chez le personnage de Condé est aussi l'exclusion sociale.

Dans cette posture, le personnage fait l'objet d'un rejet socioaffectif au point tel qu'il se sent mis au ban de la famille, d'une façon ou d'une autre. Tel est le cas pour Reynalda qui ne s'épanouit dans la famille de Gian Carlo Coppini où travaille sa mère comme domestique dès qu'ils foulent le sol de La Pointe. Après le constat de la relation adultérine qu'entretient Gian, homme marié, avec sa mère, Reynalda fait un choc psychologique. Dépourvue d'amour parental et de considération au sein de cette famille d'accueil, elle quitte la maison et tente de se suicider par noyade. Sa fille Marie-Noëlle connaît le même sort en France où sa mère la rappelle. Obligée de gérer une dure cohabitation, elle partage sa vie avec sa mère et Ludovic, un musicien expatrié. Elle qui naguère était adorée de sa mère doit désormais vivre dans la promiscuité avec son beau-père Ludovic, son frère cadet Garvey et sa mère. Cette dernière préparant une thèse de Doctorat, elle n'accorde plus de temps à sa fille une "attention particularisée car, tout compte fait, même si elle se soucie de ses enfants, elle ne peut leur donner ce qu'elle n'a jamais reçu elle-même" (Condé, 1997: 72). Il importe donc de démêler l'écheveau qu'est la quête identitaire chez le personnage de Condé.

\subsection{La quête d'identité: entre exorcisation et enjeux}

La définition du concept d'identité ne fait l'unanimité dans le monde de la science. Polysémique à souhait, il arrache à Amin Maalouf cet aveu qui résulte de son expérience de théoricien: “une vie d'écriture m'a appris à me méfier des mots. Ceux qui paraissent les plus limpides sont souvent les plus traitres. L'un de ces faux amis est justement 'identité"' (1998: 15). Malgré la difficulté qu'impose la sémantique de la notion d'identité, certains critiques la perçoivent comme "la construction progressive, par le biais de différents processus intégrateurs (ou de socialisation) qui jalonnent l'expérience individuelle, d'un système relativement cohérent de représentations de soi et de son inscription sociale (rôles statuts ou position)" (Praile, 1996: 23). Dans l'œuvre éponyme Le briseur de rosée, Edwige Danticat met en scène un personnage qui refuse d'être photographié, afin que l'image des cicatrices qu'il porte sur le visage ne lui rappellent plus son passé sombre de tortionnaire (tonton macoute) sous les Duvalier. De même dans Desirada, c'est le rejet de Marie-Noëlle, à la fois en Occident où elle négocie son intégration, qu'en terre natale où elle se sent trahie par son statut de fille naturelle, qui la résout à quitter le pays natal à la fleur de l'âge. Son processus de socialisation 
appelle à l'endurance et à l'autodétermination, tant ce personnage entend se forger une identité passe-partout qui lui ouvrirait toutes les portes sans qu'elle n'ait à s'identifier par rapport à un seul desdits espaces. Autant croire que l'identité, “ce n'est pas un vêtement égaré que l'on retrouve et que l'on endosse avec plus ou moins de grâce. [Marie-Noëlle] pourrait faire ce qu'elle voulait, elle ne serait plus jamais une vraie Guadeloupéenne" (Condé, 1997: 172).

Selon Stéphane Breton, "l'identité de l'individu se dessine d'autant plus clairement que celui qui l'exprime par la monstration de soi, puisque ce qui est montré fait autorité" (2005: 182). En d'autres termes, l'identité est un “ensemble de critères, de définitions d'un sujet et un sentiment interne. Ce sentiment d'identité est composé de différentes sentiments: sentiment d'unité, de cohérence, d'appartenance, de valeur, d'autonomie et de confiance organisés autour d'une volonté d'existence" (Mucchielli, 1986: 17). Cette assertion, tout comme la première, invitent à interroger la nouvelle personnalité de l'individu dont l'identité a subi des mutations au cours de son parcours sur le sol étranger. Le parcours des personnages de Condé oblige à asserter que l'identité n'est ni totalement figée ni définitivement permanente, puisque "les identités se forgent" (Condé, 2006: 213). Aussi, en mettant son personnage dans un mouvement permanent, la romancière guadeloupéenne entend-elle projeter à la face du monde "des parcours de vie consumés par le désir irrépressible de se lire autrement" (Abouga, 2012: 200). Se faire lire autrement semble justifier pourquoi Condé farfouille dans les mémoires dans l'optique de combler ce qu'Abouga qualifie de blancs identitaires. Cette stratégie de reconstruction identitaire des personnages par le biais de la narration des itinéraires erratiques de vies suffisamment compliquées dès le départ, positionne l'écrivaine de la Guadeloupe comme "une écrivaine du décentrement" (Abouga, 2012: 201). Dans le cas de Marie-Noëlle, il ne s'agit pas simplement d'exhiber son étiquette d'enseignante, encore faut-il faire prévaloir ce qu'induit et génère ce statut neuf à travers un rendement professionnel honorable. Le mode d'agir de Marie-Noëlle au plan académique charrie cette façon de faire. Car, après une quinzaine d'années d'étude dans son nouvel espace de vie, elle devient Professeur d'Université. L'expérience qu'elle aura engrangée l'a transformée au fil du temps. Elle apparaît au bout du compte comme une femme compétence et convaincante, de par ses savoir-être, savoir-vivre et savoir-faire.

Lorsqu'il théorise la déconstruction, Jacques Derrida affirme: “rien n'est déterminé d'avance'", pour traduire l'idée qu'aucune race, culture ou ethnie ne possèderait une identité préétablie. Point n'est donc besoin d'envisager un essentialisme identitaire qui définirait à l'avance les conduites humaines. Au contraire, l'homme, en tant qu'entité responsable, se veut maître de son devenir. Voilà pourquoi il convient de se résoudre à l'idée que "l'homme est seulement, non seulement tel qu'il se conçoit, mais tel qu'il se veut, et comme il se conçoit après l'existence, comme il se veut après cet élan vers l'existence; l'homme n'est rien d'autre que ce qu'il se fait" (Sartre, 1970: 43). Le personnage de Condé s'affilie à cette ligne de pensée. Pour

1 Dans “Déconstruction", [consulté le 14 avril 2020] microsoft@Etude2007[DVD]M.corporation.20. 
cette écrivaine, la déconstruction raciale se trame à l'aune d'une reconfiguration des notions d'appartenance et d'identité, à travers une réconciliation avec l'autre et avec soi-même. Dans cette optique, l'école nouvelle s'avère un tremplin pour l'intégration des valeurs universelles.

Dans L'Aventure ambiguë, La Grande Royale souligne l'importance d'aller à l'école que Cheikh Hamidou Kane considère, métaphoriquement, comme une guerre. Dans sa vision, elle avalise l'argument que "l'école est une forme de guerre [...] que font ceux qui sont venus, et il faut y envoyer notre élite, en attendant d'y pousser tout le pays" (1961: 47). Le roman de Condé laisse voir un penchant des personnages pour l'éducation en tant qu'elle participe du développement individuel et collectif. Appelant une forme d'universalisme, l'école impulse un mode d'être incarné par Marie-Noëlle dans le récit condéen. Immigrée aux EtatsUnis, elle s'aperçoit que l'Amérique est une terre où règne le cosmopolitisme. Elle découvre, par ailleurs, que la ville de Boston ouvre la voie à un syncrétisme culturel impressionnant. Le narrateur retrace ainsi son itinéraire: "elle allait et venait dans un tout autre monde. Parmi des Noirs, des Blancs, des basanés, des métis, des métèques, des exilés, des transplantés, des déracinés" (Condé, 1997: 121).

Par-delà le décryptage de l'ancrage historique du récit de Maryse Condé, une seconde étape s'impose, qui touche à la question de l'esthétique à l'œuvre dans la narration des faits évoqués.

\section{L'aventure de l'écriture de l'immigration}

Dans son déploiement épistémologique, le sociocritique vise le sociotexte, c'est-à-dire "le statut du social dans le texte et non le statut social dans le texte" (Reynove, 1979: 107). Nnomo Zanga et Messina expriment mieux l'avis de Reynove en reprécisant l'interaction féconde qui lie texte et art littéraire. Ils affirment que: "la représentation de la réalité n'est pas réductible à ce qui en est dit uniquement; elle est également présente dans la façon de le dire, dans le matériau dont on sert pour le dire" (2014: 37). Nous empruntons à Jean Ricardou sa célèbre assertion "le roman est davantage une aventure de l'écriture qu'une écriture de l'aventure" (1967: 24), pour traduire dans les faits cette synergie opérante dans le phénomène de la création littéraire. Car, si la société crée la littérature, celle-ci la crée aussi, suivant un processus recréant qui replace le démiurge au centre de l'acte même de création. Ainsi, parler de fiction c'est mettre en équation, "le niveau de l'invention et de l'agencement de l'histoire" (Genette, 1991: 17). L'art de Condé a pour piliers l'intergénéricité, l’usage des modes de liaison.

\subsection{L'écriture hybride et l'art du souvenir: intergénéricité et analepse}

L'esthétique du roman africain n'est parfois pas différente de celle observée dans le roman guadeloupéen. On y voit inscrite l'intergénéricité qui traduit une forme d'hybridité 
dont le mélange des genres s'avère l'une des caractéristiques les plus significatives. Pour Josias Semujanga, la question des genres constitue un item heuristique polémique qui met de l'avant le problème de l'intergénéricité. Il s'agit des "diverses formes d'interaction entre les catégories génériques, canoniques ou non. L'intergénéricité étudie, en effet, les processus de production de sens provoqués par l'union ou l'affrontement de deux ou plusieurs genres, par l'entremise de stratégies diverses" (2018: 213). S'appuyant sur les travaux de Robert Dion, Frances Fortier et Elisabeth Haghebaert, François Harvey identifie trois processus d'interaction générique: la différenciation, l'hybridation et la transposition. Celle qui nous intéresse dans le cadre de cette étude est l'hybridation. Elle se présente comme "la combinaison de traits génériques hétérogènes dans une même œuvre" (2011: 128).

De l'avis d'un autre critique, le phénomène de l'intergénéricité épouse les caractéristiques d'une œuvre baroque. Il affirme qu'elle "réagit contre l'idée de modèle générique. Elle réunit en elle plusieurs tons et genres différents. Le roman y côtoie la poésie, la chanson, la tragédie ou la comédie dans une fusion libre et totale des formes esthétiques" (Semujanga, 2018: 214). Cette modalité d'écriture, inhérente à la création littéraire baroque, s'avère d'autant plus opérante qu'elle définit une marque de l'esthétique condéenne. Car, à la vérité, "on ne saurait saisir les enjeux de l'écriture de M. Condé sans tenir compte de son refus de tout enfermement esthétique" (Abouga, 2012: 212). Dans cette perspective, le phénomène de l'hybridisme chez Condé se manifeste à travers l'usage de la pseudo-chronique. Certes, l'ensemble du récit ne fonctionne pas sous le régime de l'alternance entre les genres, même si l'onirique intervient de temps à autre dans l'histoire par le biais des rêves de Marie-Noëlle. Quatre dates déclinées sous la forme semi-complète de jour-mois-année sont évoquées dans la trame romanesque. Leur évocation rend compte des mutations morales, psychologiques et intellectuelles que traverse le personnage principal lors de ses pérégrinations. Il apparaît que Marie-Noëlle est née un mardi gras (février-mars), le "05 juillet 1960 à La Pointe en Guadeloupe" (Condé, 1997: 14). Cette date rappelle un triste souvenir à cette fille parce qu'elle réfère au jour que sa mère Reynalda Altamira l'a abandonnée peu après sa venue au monde. On sait également que Reynalda avait 15 ans comme le révèle l'indice: "dont le bassin de quinze ans" (Condé, 1997: 14), puisqu'elle est née en 1945, “...à la fin de la Deuxième Guerre, l'année même où je naissais de père inconnu à la Désirade" (Condé, 1997: 67). Marie-Noëlle a donc dix ans à la date du "05 juillet 1970" (Condé, 1997: 14), tandis que Reynalda en a vingt-cinq en 1970. Elle intègre la famille Coppini à La Pointe le "10 septembre 1955" (Condé, 1997: 68).

Alors que Marie-Noëlle vient de célébrer l'anniversaire de sa décennie et entre en classe de sixième, sa marraine reçoit une lettre de Reynalda réclamant sa fille en France. Le départ pour l'hexagone, le "31 octobre 1970" (Condé, 1997: 33), apparaît comme le début d'une odyssée qui prend l'allure d'une quête de la famille et de l'identité. En juin 1978, elle obtient son baccalauréat et va aux États-Unis. Roman sur la question de l'immigration, $D e$ - 
sirada se singularise, au total, par cette migration temporelle à travers le temps qui passe et qui laisse une marque indélébile sur la vie de Reynalda et de sa fille entre 1960 et 1978. Le passage récurrent d'un espace de vie à un autre participe de la dynamique de construction d'une forme de nomadisme au plan scriptural. Abouga affirme dans cette optique que "la pratique du nomadisme chez M. Condé devient donc une nécessité. Nécessité de voyager, de fuir les failles de la civilisation occidentale, la désagrégation de ses idéaux et de ses principes, d'observer le monde, de l'enregistrer et de l'emmagasiner afin de se construire' (2012: 211).

On sait donc, au regard des indicateurs de datation, que la migration temporelle s'étale sur dix-huit ans exactement. Cette plongée constante dans le passé et ce rejaillissement dans un temps spectaculairement mutant, se laissent saisir à travers la figure de l'analepse, dans le métalangage de Genette. Il s'agit de “l'évocation après coup d'un événement antérieur au point de l'histoire où l'on se trouve" (1972: 82). L'anachronie de type analeptique permet de ressasser les temps forts de l'histoire de la mère de Reynalda. En tant qu'elle participe du brouillage temporel instauré par le narrateur omniscient, l'analepse annonce les signes avant-coureurs de l'incertitude que présage son aventure. On peut dès lors convenir avec Claude Roy que l'œuvre littéraire est "l'histoire que n'ont jamais écrit les historiens" (1967: 43). À l'instar de la datation, les modes de liaison favorisent également la mise en texte de la diégèse.

\subsection{Les modalisations de la liaison}

Trois stratégies d'écriture modalisent le phénomène de la liaison dans un roman: 1'alternance, l'enchainement et l'enchâssement. L'alternance renvoie au procédé par lequel on raconte deux ou plusieurs histoires en interrompant tantôt l'une tantôt l'autre pour la reprendre à l'interruption suivante. Aussi, à l'intrigue principale structurée autour de macro-récits, se greffent de micro-récits qui apparaissent comme des récits secondaires servant de témoignage ou d'illustration. Chez Condé, les épisodes six de chaque partie du roman relatifs respectivement au récit de Reynalda, de Nina et de Ludovic constituent des formes d'alternance. Contrairement à l'enchainement où "les épisodes se suivent dans l'ordre I, II, III" (Goldenstein, 1999: 99), l'enchâssement se révèle au lecteur sous une autre figure. Il s'offre “lorsqu'un récit s'insère dans la trame d'un récit initial, lorsqu'une histoire est introduite dans une autre histoire, il y a enchâssement et les épisodes se suivent dans l'ordre I, II, I " (1999: 99). Il ne s'agit donc plus d'un entrelac d'histoires, de sorte que l'intrigue se compose d'un ensemble de petits récits qui fusionnent aux fins de former un grand ensemble composite. La technique de l'enchâssement chez Condé se voit à travers le récit de Reynalda qui est recoupé dans le rêve de Marie-Noëlle. Présenté de manière asymétrique sous forme de rêve dans le macro-récit de la vie de Reynalda, ce micro-récit peut être représenté conformément 
au découpage en Parties I-II-I, proposé par Jean Pierre Goldenstein. Les parties assurant la liaison par le biais de l'enchâssement sont les suivantes:

Partie I: elle renvoie à l'épisode six (6) du roman: quelques mois après l'arrivée de Marie-Noëlle en France, sa mère Reynalda commence à lui relater l'histoire de sa vie depuis la Désirade jusqu'à La Pointe chez la famille de Gian Carlo Coppini (Condé, 1997: 61-70). Partie II: elle renvoie à l'épisode sept et huit (7-8) du roman. Le narrateur rebondit sur le récit des circonstances du départ de Marie-Noëlle pour les Etats-Unis et recoupe la suite du récit précédent sous la forme du rêve de Marie-Noëlle (Condé, 1997: 71-84). Partie I: elle renvoie à l'épisode neuf (9) du roman Desirada lorsque Reynalda continue son histoire et que Marie-Noëlle retourne en France après la naissance de sa sœur cadette Angéla (Condé, 1997: 85-101). Un commentaire de type analytique permet de relever l'hétérogénéité des deux récits. Car, alors que la Partie I amorce une histoire rocambolesque, symbole d'une retrouvaille entre mère et fille, la Partie II crée un brouillage qui dissout la retrouvaille par un voyage diluvien avant de revenir à la Partie I. L'interposition de la Partie II tronque inégalement la Partie I. Elle engendre une anachronie narrative au plan de la structuration du récit, et, au plan sémiologique, une brouille émotionnelle entre la mère et sa fille du fait de la rupture observée dans la linéarité de la diégèse. Le récit de Condé ouvre des perspectives qui sont en réalité autant de leçons qui constituent en réalité ce que Mitterand qualifie de discours sur le monde.

\section{L'immigration: entre discours doctrinal et discours sur le monde}

Le discours sur le monde dans le jargon mitterandien, correspond à l'idéologie que défend un auteur à travers son texte. Jean Marc Mourra affirme qu'en littérature, l'idéologie ou le discours doctrinal est "capturé par le jeu du sentiment et de l'imagination, [il] réfracte d'une manière spécifique, $[\ldots]$ une nouvelle manière singulière de penser et d'exprimer le monde, c'est trop évident" (1999: 12). Maryse Condé construit son discours en tissant les fils constitutifs d'une nouvelle identité humaine. Celle-ci se génère à travers l'itinéraire existentiel des personnages et les enjeux éthiques qui sous-tendent les mouvements migratoires.

\subsection{L'itinéraire existentiel des personnages}

Il va sans dire, au regard de la culture livresque y relative, que l'itinéraire de survie qu'arpente l'immigré est jonché d'obstacles. Les ressorts d'une telle vie de surpassement continuel sont le travail indécent et le choc psychologique.

La souffrance, pourrait-on dire, est pour l'homme une école de la vie. Si d'une part, cette assertion révèle l'essence de la vie de l'homme, à savoir la souffrance, elle interpelle davantage sur une prise de conscience personnelle des individus afin que ces derniers changent 
d'option de vie lorsque le rêve d'errer les hante. S'agissant par exemple du travail indécent, Marie-Noëlle exerce comme femme de ménage aux États-Unis; elle y berce Molara, la fille adoptive d'Anthéa Jackson. Il s'agit d'un emploi précaire qui génère un salaire dérisoire. Alexie Tcheuyap souscrit à cette allégation en mettant en exergue un certain gâchis qui découle de l'acte obstiné de partir ailleurs. Relevant les limites d'une démarche migratoire vouée à l'échec du migrant au plan financier, il déclare: “au terme d'une émigration vers un ailleurs espéré meilleur, les diplômes acquis après un dur labeur perdent souvent toute leur valeur et conduisent moins souvent au succès qu'à l'alcoolisme, aux trafics de tous genres, [...] ou au sang des boucheries industrielles" ${ }^{20}$. Hormis le sport et la musique qui offrent à l'immigré de réels espoirs en termes de gain, l'indécence devient l'activité favorite à laquelle il se livre. Elle affecte pour ainsi dire son quotient psychologique.

Au plan psychologique, le personnage de Condé, qui baignait dans la nostalgie en opérant sans cesse un retour dans l'imaginaire, rompt désormais toute volonté d'introspection. Marie-Noëlle met fin aux enquêtes visant à retrouver son père. Ce faisant, elle finit par s'accepter tel qu'elle est en confessant: "je me tairai donc en attendant qu'à mon tour, j'apprenne à inventer des vies" (Condé, 1997: 281). Son parcours initiatique lui confère une certaine expérience, dès lors que par le travail elle reprend goût à la vie. D'ailleurs, c'est l'amour pour le travail qui devient la raison de vivre de Reynalda à un moment de sa vie. Cette dernière entreprend d'inculquer cette vertu à Marie-Noëlle afin de parachever son processus de maturation. Le travail libère l'esprit des pesanteurs liées à l'immigration comme le révèlent ces mots d'une Reynalda plus allègre à l'adresse de Marie-Noëlle:

- C'est le travail qui m'a mise là où je suis là. Dans un bureau au premier étage de la mairie, avec un secrétaire à ma dictée. C'est le travail et pas autre chose. Aujourd'hui, je ne connais ni maître ni maîtresse. Je fais ce que je veux, comme je veux, quand je veux. Pendant des années, les gens m'ont traitée comme un chien [...] C'est bien fini. Il faut décider soi-même parce que personne ne vit votre vie à votre place (Condé, 1997: $62)$.

Au plan moral, le travail a contribué à façonner la personnalité des héroïnes de Condé au regard des parcours qu'elles ont effectués et des influences qui se sont opérées dans leur vie. Reynalda et Marie-Noëlle peuvent, dès lors, s'estimer émancipées, en tant qu'elles se positionnent somme des actrices de l'autonomisation de la femme. La preuve en est que Marie-Noëlle met à profit les conseils maternels et devient professeur d'université. Autant partager l'avis de Salie qui, dans Le ventre de l'Atlantique, allègue que chaque miette de vie doit servir à conquérir la dignité. Cette conquête se dévoile aussi à travers le décryptage des enjeux éthiques que recèle l'immigration dans le roman de Condé.

$2<$ http://www.Uwa.Edu./mots pluriels/MP2002 édito. 1 html> [consulté le 15 avril 2020]. 


\subsection{Des enjeux éthiques de l'immigration}

Quête inlassable de soi-même à travers les expériences de vies compromises hors des limites du terroir du migrant, l'immigration apparaît néanmoins comme une entreprise bénéfique de l'avis de certains observateurs. Henri Lopès conforte ce point de vue lorsqu'il déclare: “j’y ai appris le monde jamais nulle part auparavant, je m'y suis enrichi. J'ai regardé au fond de moi et j'ai mieux saisi mon pays. C'est là-bas que j'ai compris la fécondité de la solitude pour apprendre, réfléchir, créer" (1992: 102). Si d'une part, Lopès entend ressortir les bons côtés de l'immigration, force est de relever que cette pratique, surtout clandestine, reste une grave menace à l'épanouissement des individus épris d'aventures. Dans Un amour sans papier, l'ailleurs, symbolisé par le France, présente une double facette mitigée: “d'une part celle d'une nation riche et triomphante, et de l'autre celle des banlieues difficiles, celle de la kyrielle des sans-emplois, sans logis, sans ressources, sans-papiers" (Etoke, 1999: 56). Au regard de l'argument qui précède, force est de reconnaître que l'immigration chez Condé soulève des enjeux éthiques diversifiés.

L'éthique, faut-il le rappeler, “est autant une affaire de comportement qu'un ensemble de compétences et de connaissances” (Pipper, Gentile \& Daloz Parks, 1993: 115). L'enjeu comportemental est donc le fondement de l'éthique dans la trame condéenne. L'immigration hasardeuse plonge le sujet migrant dans un sentiment de solitude. Du fait de son acculturation, l'immigré se dépersonnalise et s'extrovertit en vue d'embrasser la culture de l'autre. En ce sens, on peut affirmer que "la solitude est une tyrannie de l'identité. Si elle était quelqu'un d'autre, le hasard décidera[sic], une femme connaîtra un peu de paix, d'équilibre. On éprouve toujours de l'ironie à devenir qui on n'est pas" (Bosquet, 1992: 474). Chez Condé, le sentiment de solitude qui agite les personnages résulte de leurs mauvaises conditions d'accueil par les Occidentaux. Ces derniers n'intègrent pas toujours dans leurs modes de vie certaines valeurs humanistes antillaises telles que les salutations fraternelles, la sympathie, le visites et autres, la convivialité. Le souvenir de la vie menée au pays natal induit chez certains migrants africains par exemple un retour au pays. Mais il convient de souligner que pour les Antillais, cette question du retour au pays est plus complexe. L'indice de retour au pays natal se mesure à l'aune des souvenirs de l'île, contrairement au pays d'origine des Africains qui, lui, correspond au pays des ancêtres. La manifestation du désenchantement instaure une option de vie construite autour de l'idée que "le salut est possible hors de l'immigration" (Diome, 2001: 71). Mais Diome nuance ses propos en invitant l'immigré à entreprendre un mouvement de va et vient qui lui assure respect et considération autant dans la terre d'accueil qu'à son retour au pays. Elle précise bien que: “je ne veux pas dégoûter les nôtres de l'Occident, mais il faut prendre conscience du fait qu'il faut y aller autrement et mettre tous les atouts de son côté avant de partir" (Diome, 2003: 71). C'est le cas de dire que l'immigration présente dans certains cas des facettes multiples. Elle est certes périlleuse pour certains mais participe 
de la reconstruction identitaire pour d'autres. Elle leur permet non seulement de se réinventer, mais aussi et surtout de profiter des bienfaits qu'offrent l'exotisme et le tourisme. Maryse Condé milite en faveur d'une nouvelle philosophie de la vie dans le cadre des rapports NordSud en pointant du doigt les inégalités sociales, le déterminisme, le conformisme, le racisme et le rêve illusionniste. Promotrice de l'humanisme, apôtre de l'existentialisme et de l'altérité, l'écrivaine de la Guadeloupe invite à transcender les identités ataviques.

\section{Conclusion}

Au total, le roman de Maryse Condé s'avère un espace de modélisation d'un nouveau type d'immigré. Ce dernier peut construire, par son travail et l'effort soutenu, un projet de vie abouti, par-delà ses échecs successifs et son implication dans un processus de nomadisation riche en péripéties existentielles: irresponsabilité parentale, solitude et choc psychologique. La nouvelle identité postulée par la Guadeloupéenne se voit à travers un double prisme qui s'avère somme toute problématique: l'idéalisation exacerbée de l'Occident et l'exorcisation des pratiques culturelles rétrogrades. Pour construire son avenir, il importe pour l'immigré de dépasser le cadre d'un passé sclérosant en vue de susciter de nouvelles valeurs fondées sur les vertus de la tolérance, de la liberté et de l'altruisme. Si la volonté de réussir son intégration dans l'espace occidental induit l'adoption par l'immigré des valeurs civilisationnelles étrangères, force est de dire que l'acceptation de l'autre et son intégration dans un milieu de vie qu'il ne connaît pas se révèlent indispensables dans la promotion de l'humanisme que la romancière appelle de tous ses vœux. Son œuvre de fiction apparaît, dès lors, comme une tribune où elle lance un vibrant appel à tous les gestionnaires du pouvoir politique. Elle les convie à un management conséquent, plus rationnel, ou tout au moins plus juste et plus équitable dans la postulation de la bonne gouvernance. C'est dans cette plateforme institutionnelle de gestion des affaires de la cité que se créeront des emplois, lesquels dispenseront les plus jeunes de s'engager dans des migrations fantaisistes et parfois suicidaires et hasardeuses auxquelles ils se livrent continuellement. Maryse Condé voue par contre un culte au travail et à l'effort permanent, qui, seuls, ouvriront la voie du succès de demain à un migrant sur le sol étranger. La capacité du démiurge guadeloupéen à portraiturer un nouveau visage de l'immigré en fait un acteur intrépide qui redéfinit sa propre identité en s'affranchissant des pesanteurs qui en obère l'éclosion. Autant croire que le démiurge Condé se positionne comme une promotrice de la diversité identitaire car enfin, "il ne s'agit pas de changer une identité pour une autre, mais plutôt de les accueillir et de les adopter. Ce processus fait sa richesse, surtout quand les différentes identités sont assumées dans la sérénité et non dans le déchirement, ni dans la dégradation de la mémoire" (Bouraoui, 2005: 9). 


\section{Références bibliographiques}

ABouga, Yvette Marie-Edmée. 2012. "Migrations des imaginaires: traversées des milieux et des mémoires dans Victoire, les saveurs et les mots de Maryse Condé", in Revue Ecritures. Littérature et migrations dans l'espace francophone, $\mathrm{n}^{\circ}$ XI, 199-213.

ApriLe, Sylvie \& Stéphane, Duforx. 2009. Les mots de l'immigration. Paris, Belin.

Attali, Jacques. 2003. L'homme nomade. Paris, Fayard.

BosQuet, Alain. 1992. Les solitudes. Paris, Gallimard.

Bouraour, Hedi. 2005. Transpoétique: éloge du nomadisme. Montréal, Mémoire d'encrier.

Breton, Stéphane. 2005. Télévision. Paris, Grasset et Fasquelle.

Chevrier, Jacques. 1989. Anthologie africaine d'expression française. Paris, Hatier.

Conde, Maryse. 2006. Victoire, les saveurs et les mots. Paris, Mercure de France.

Conde, Maryse. 1997. Desirada, Paris, Robert Laffont.

Danticat, Edwige. 2005. Le briseur de rosée. Paris, Grasset.

Diome, Fatou. 2001. La préférence nationale. Paris, Présence Africaine.

Diome, Fatou. 2003. "Interview" in Amina, n 403, 13-14.

Dolby, Nadine. 2006. "Popular Culture and Public Space in Africa: The Possibilities of Cultural Citizenship" in African Studies Review, volume 49, number 3, 34-45.

Eтоке, Nathalie. 1999. Un amour sans papier. Paris, Cultures croisées.

Genette, Gérard. 1972. Figures III. Paris, Seuil.

GenetTe, Gérard. 1991. Fiction et diction. Paris, Seuil.

Goldenstein, Jean Pierre. 1999. Lire le roman. Bruxelles, Duculot.

Goldmann, Lucien.1964. Pour une sociologie du roman. Paris, PUF.

Grand Dictionnaire Encyclopédique Larousse. 1982. Paris, Larousse.

Lopes. Henri. 1992. Sur l'autre rive. Paris, Seuil.

Kane, Cheikh Amidou. 1961. L'aventure ambiguë. Paris, Julliard.

MaAlouf, Amin. 1998. Les identités meurtrières. Paris, L'Harmattan.

Mitterand, Henri. 1980. Le discours du roman. Paris, Seuil.

Mourra, Jean Marc. 1999. Littérature francophone et théorie postcoloniale. Paris, PUF. 
Anales de Filología Francesa, n. ${ }^{\circ}$ 28, 2020

Pierre Suzanne Eyenga Onana

Mucchielli, Alex. 1986. L’identité. Paris, PUF.

Nnomo Zanga, Marcelline \& Gérard-Marie Messina. 2014. Pour une critique du texte négro-africain. De la sociocritique à la politocritique. Paris, L'Harmattan.

Pipper, Thomas, Mary Gentile \& Sharon Daloz Parks. 1993. Enseigner l'éthique? Le Havard Business School relève le défi. Boston, Havard Business School Press.

PraIle, David. 1996. L'homme naufrage. Des représentations identitaires chez les jeunes hommes issus de l'immigration maghrébine. Louvain-la-Neuve, UCI.

Reynove, Jean. 1979. Sémiotique. Paris, PUF.

Ricardou, Jean. 1967. Problèmes du nouveau roman. Paris, Seuil.

Roy, Claude. 1967. Défense de la littérature. Paris, Gallimard.

Semujanga, Josias. 2018. "De l'intergénéricité comme forme de baroque dans le roman de Sony Labou Tansi” in Jean CLeo Godin, Nouvelles écritures francophones, 202-215.

TCHEUYAP, Alexie. <http://www.Uwa.Edu./motspluriels/MP2002 édito.1html> [15/4/2020]. 
